the cardiotoxicity of ketamine and the protective effects of metoprolol.

Methods Rats and rabbits were divided into control, ketamine poisoning and ketamine plus metoprolol groups. Ketamine (40 $\mathrm{mg} / \mathrm{kg} /$ day, intraperitoneally) and metoprolol $(20 \mathrm{mg} / \mathrm{kg} /$ day, orally) were administered continuously for 12 (rats) or eight (rabbits) weeks, respectively. Cardiac function and electrophysiological disturbances were examined, collagen volume fraction and apoptotic cell number were analysed and remodelling related proteins were detected by immunohistochemistry and western blot.

Results Rabbits showed decreased left ventricular ejection fraction, slowed ventricular conduction velocity, and increased susceptibility to ventricular arrhythmia. Metoprolol prevented these pathophysiological alterations. In ketaminetreated rats, cardiac collagen volume fraction and apoptotic cell number were much higher than normal, which were mitigated by co-administration of metoprolol. Consistently, the expressions of poly (ADP-ribose) polymerases-1, apoptosis inducing factor and nuclear factor kappa-light-chain-enhancer of activated B cells were all increased after ketamine treatment, and sharply reduced after metoprolol administration. Moreover, ketamine enhanced sympathetic sprouting manifested by increased growth-associated protein 43 and tyrosine hydroxylase expression, which were also prevented by metoprolol.

Conclusion Long-term abuse of ketamine caused significant ventricular myocardial apoptosis, fibrosis and sympathetic sprout, which altered the electrophysiologic property of the heart and increased the susceptibility to malignant arrhythmia that may lead to sudden cardiac death. Metoprolol prevented the cardiotoxicity of ketamine, representing a promising therapeutic strategy. [gw22-e0288]

\section{KETAMINE-INDUCED VENTRICULAR STRUCTURAL, SYMPATHETIC AND ELECTROPHYSIOLOGIC REMODELLING: PATHOLOGICAL CONSEOUENCES AND PROTECTIVE EFFECTS OF METOPROLOL}

Li Yue', Shi Jing ${ }^{1}$, Han Chunliu', Li Weimin'1, Yang Baofeng ${ }^{2}$, Pan Zhenwei², Dong Deli², Liu Guangzhong', Sheng Li', Liu Lei', Sun Danghui', Tan Xiangyang', Gong Zhihua1, Gong Yongtai' 'The First Clinical Hospital, Harbin Medical University, Harbin, China; ${ }^{2}$ Department Of Pharmacology And Bio-pharmaceutical Key Laboratory Of Heilongjiang Province And State Harbin Medical University, Harbin, China

10.1136/heartjnl-2011-300867.18

Purpose Growing evidence suggested long-term abuse of ketamine did harm to the heart and increased the risk of sudden death. The present study was performed to explore 